\title{
Long-Term Mortality and State Financial Support in Invasive Meningococcal Disease-Real-World Data Analysis Using the French National Claims Database (SNIIRAM)
}

Jing Shen · Stéphane Bouée · Emmanuel Aris · Corinne Emery •

Ekkehard C. Beck

Received: June 29, 2021 / Accepted: October 1, 2021 / Published online: November 17, 2021

(C) GlaxoSmithKline Biologicals SA 2021, corrected publication 2022

\section{ABSTRACT}

Objectives: Invasive meningococcal disease (IMD) is an uncommon disease known for its acute phase mortality and long-term sequelae. The objective was to assess the impact of IMD on post-discharge mortality risk and dependence on the French state for financial aid.

Methods: A 6-year retrospective analysis in the national insurance database (SNIIRAM) assessed mortality in IMD cases (both during acute phase and post-discharge) and matched controls as well as benefit claims (i.e., for salary loss compensation [SLC], long-term sickness [ALD] and complementary health insurance [CMUc]). Observed survival data were extrapolated to estimate lifetime life expectancy following IMD. Results: Between 2012 and 2017, 3532 incident IMD cases were hospitalised in France (peak in $<2$ years and 15-24 year olds), of which $23.3 \%$ developed sequelae. With an average follow-up of 2.8 years, $12.9 \%$ of cases vs. $3.2 \%$ of controls died $(p<0.0001)$, with significantly

Supplementary Information The online version contains supplementary material available at https:// doi.org/10.1007/s40121-021-00546-z.

J. Shen · E. Aris · E. C. Beck $(\bowtie)$

GSK, Value Evidence, Avenue Fleming 20, 1300

Wavre, Belgium

e-mail: ekkehard.x.beck@gsk.com

S. Bouée · C. Emery

CEMKA, Bourg-La-Reine, France more cases than controls dying both during the acute phase and post-discharge. Around a third of these deaths occurred post-discharge. Extrapolation to lifetime life expectancy estimated that having IMD at any age significantly reduces life expectancy in survivors of the acute disease phase, e.g., by around 16 years for cases aged 0-50 years. IMD cases in France were significantly more likely to receive state-funded SLC (relative risk [RR] 3.9, 95\% confidence interval [95\% CI] 2.3-6.4) and ALD benefits (RR $1.85,95 \%$ CI 1.71-2.00).

Conclusions: IMD has a significant impact on mortality post-discharge, expected to persist over a lifetime. In addition to long-term sequelae, the financial burden extends beyond the healthcare sector. These results highlight the importance of IMD prevention (e.g., vaccination).

\section{PLAIN LANGUAGE SUMMARY}

Invasive meningococcal disease (IMD) is an uncommon disease mainly affecting children, with severe consequences such as a risk of dying within hours of symptoms and a risk of developing long-term conditions affecting health, learning and ability to work. Little is known of the risk of dying in survivors after discharge from hospital or of survivors' financial support needs. The French national insurance claims 
database (SNIIRAM) was reviewed for data on IMD patients hospitalised between 2012 and 2017 and matched controls without IMD. Data, available following IMD hospitalisation for an average of around 3 years, were extrapolated to estimate the lifelong impact of the disease. Among 3532 hospitalised IMD cases, the study found that nearly 13\% died, of which a third of deaths occurred post-discharge. The cases who survived the acute disease phase were also more likely to require government funds because of loss of salary or to cover long-term healthcare costs. In addition to the well-known acute phase burden of IMD, this study has shown that there is a long-term effect on risk of dying and on need for government support. This demonstrates the importance of prevention, for example, by vaccination.

Keywords: Invasive meningococcal disease; Long-term mortality; Financial aid; France

\section{Key Summary Points}

\section{Why carry out this study?}

Invasive meningococcal disease (IMD) in an uncommon but severe infectious disease, primarily affecting young children and adolescents.

The risk of death in the acute phase and of long-term disability in survivors of the acute phase is well recognised.

This study assessed the long-term risk of death in IMD survivors and their need for state-provided financial assistance.

\section{What was learned from the study?}

Real-world evidence in France over a 6-year period found that $12.9 \%$ of IMD cases died, with a third of deaths occurring post-discharge. Extrapolation of survival data over lifetime predicted that IMD significantly reduces life expectancy (by 16 years for cases aged $0-50$ years old).
IMD survivors in France were nearly four times more likely to need state-funded benefits because of loss of salary and nearly two times more likely to need benefits to cover healthcare costs for a long-term disability compared to controls.

IMD has a significant long-term effect on mortality and places a financial burden on the state beyond healthcare costs.

Prevention of IMD through vaccination is of vital importance.

\section{INTRODUCTION}

Invasive meningococcal disease (IMD) caused by Neisseria meningitidis infection is an uncommon but severe disease, typically manifesting as meningitis and/or septicaemia [1]. The highest incidence is in the most vulnerable age group of infants and young children, followed by adolescents [2]. IMD patients can deteriorate rapidly, leading to high case fatality rates of $8-15 \%$ during initial hospitalisation in European countries [3], with higher case fatality rates in older age groups (15\% in young adults to $30 \%$ in 75 year olds [4]) and significant 50\% case fatality rates in untreated cases [5]. In addition, around $30 \%$ of survivors of the acute phase of the disease are discharged with one or more sequelae, and $10 \%$ with long-term or disabling sequelae, such as hearing loss, cognitive impairment, skin scars and amputations [6].

Although IMD is uncommon, the disease burden is high with a long-term impact on the patient, their family and society [7]. IMD remains a public health concern, with high costs to the healthcare system and risks of outbreaks due to its unpredictable epidemiology [8-10]. Several European countries recommend childhood vaccination with vaccines conferring protection against serogroup $B$, serogroup C and/or serogroups ACWY IMD [11, 12]. Of the five serogroups that cause most disease worldwide $(B / A / C / W / Y)$, serogroup $B$ currently causes most cases of IMD in Europe [3], including France [13]. 
The literature on the burden of IMD tends to focus on acute phase mortality [4] or longer term medical costs and clinical outcomes [14] and has, through systematic review [15], identified a broader range of sequelae in survivors than previously reported in individual studies. There is, however, limited research on IMD survivors beyond the acute phase, in particular on the risk of death and costs beyond direct medical costs, such as the need for state financial aid (e.g., government benefits to help in case of reduced working ability or to help cover healthcare costs). The aim of this study, therefore, is to fill this gap in the literature.

For rare diseases such as IMD, it is crucial to use long-term and large data sets to capture disease impact. In France, the national public health insurance database (Système National d'Information Inter Régimes de l'Assurance Maladie-SNIIRAM) set up in 2003 now covers $99 \%$ of the population from birth to death [16] and is, therefore, an ideal database to use for this research.

\section{METHODS}

\section{Study Design and Population}

A 6-year retrospective case-control study was conducted in the SNIIRAM database. All incident IMD cases (ICD-10 diagnosis codes A39.0 to A39.9) hospitalised between 1 January 2012 and 31 December 2017 were included and followed until their death or the end of the study period, whichever occurred first. For every IMD case, three random controls were included, matched by age (to age when IMD occurred in the matching case), sex and area of residence (i.e., by French Department). The selection of the control group did not require that controls were hospitalised.

\section{Data Collection}

The SNIIRAM database contains anonymised demographic and healthcare consumption information on almost $99 \%$ of the French population from birth to death. The database collates reimbursed claims from all health insurance plans in France, from the national public and private hospital discharge database (Programme de Médicalisation des Systèmes d'Information [PMSI]) as well as the outpatient reimbursed health expenditures database (Données de consommation inter-régimes [DCIR]) and is linked to the national death registry. It also contains information on patients receiving financial benefits for long-term chronic conditions or to supplement healthcare costs for people with a low income $[16,17]$.

For this analysis, demographic data on age at time of first meningococcal hospitalisation (incident infection), date of death during study period (if applicable), sex and area of residence were collected. Hospitalisation data were used to identify incident IMD cases at their first (index) hospitalisation, after which cases were followed prospectively in the database to identify any long-term IMD complications and sequelae. Predefined potential IMD sequelae $[18,19]$ were identified through their diagnostic codes, medical procedures or treatments performed in inpatient and outpatient settings. No serogroup-specific data on IMD cases were available. Data on three types of state financial aid (i.e., provided by the government and beyond the health sector) were collected: salary loss compensation (SLC) for disability (pension d'invalidité), exemption from co-payments for long term/chronic disease (Affection de longue durée, ALD) and complementary insurance for people with a low income (Couverture Médicale Universelle complémentaire-CMUc).

\section{Analyses}

Variables were described by sample size and number of missing values, using mean, standard deviation, median, minimum and maximum values for quantitative variables and responses expressed as percentages for qualitative variables. The SAS ${ }^{\circledR}$ v9.4.0 software programme (Cary, NC, USA) was used for data management and statistical analysis.

From the observed mortality data over the 6-year study period, Kaplan-Meier survival curves were plotted for IMD cases and controls 
(for IMD cases, the analysis included acute phase and post-discharge mortality and only post-discharge mortality in survivors of the acute phase). The post-discharge survival data (observed for a maximum of 6 years) were also extrapolated to estimate life expectancy over the life course for both IMD survivors and the matched control group as a comparison, fitted using flexible parametric survival models [20]. The area under the survival curve was used to calculate life expectancy for both IMD survivors and controls.

Risk ratios (RR) were calculated to compare the number of IMD cases vs. controls receiving state financial aid (SLC due to disability, ALD and $\mathrm{CMUc}$ ).

\section{Compliance with Ethics Guidelines}

The study was conducted in accordance with the Helsinki Declaration of 1964 and later amendments and relevant international and French regulatory requirements. Patient data in the database are anonymised using an irreversible double encryption. Access to the SNIIRAM database is regulated by a Committee of Expertise for Research, Studies and Evaluations in the field of Health, to which the present study protocol was submitted for approval. Since this was a retrospective study of an anonymised database and had no influence on patient care, ethics committee approval was not required. Use of the database for this type of study is regulated by the French national data protection agency (Commission Nationale de l'Informatique et des Libertés), to which the protocol was submitted for approval.

\section{RESULTS}

Over the 6-year study period (2012-2017), there were 3532 incident cases of IMD hospitalised in France and they were followed on average for $2.8 \pm 1.9$ (median 2.8 [range 0-6.0]) years. The age groups with the highest incidence were infants and young children $(<2$ years) and adolescents and young adults (aged 15-24 years), accounting for $22.0 \%$ and $19.6 \%$ of IMD cases, respectively (Fig. 1). The median age of IMD cases was 21 (interquartile range [IQR] 4-52) years. Among IMD cases, 23.3\% (823) had sequelae reported in the database. There were 10,596 matched controls without IMD, followed for $3.1 \pm 1.8$ (median 3.0 [range 0-6.0]) years.

\section{Observed IMD Mortality Data (SNIIRAM 2012-2017)}

During the study period, IMD cases were significantly more likely to die than controls (456 IMD deaths [12.9\%] vs. 344 [3.2\%] in controls, $p<0.0001)$. IMD is characterised by a severe acute phase, during which around two-thirds of IMD deaths occurred, i.e., 8.3\% (293/3532) of cases died during the index hospitalisation. The remaining approximately one third of IMD deaths occurred post-discharge during the study follow-up, i.e., 5.0\% (163/3239 survivors of the acute phase). Among post-discharge deaths, $42.3 \%(69 / 163)$ had IMD sequelae noted in the database. The overall risk of post-discharge death during the 6-year observation period was $3.5 \%(94 / 2709)$ among survivors with no sequelae and $8.4 \%(69 / 823)$ among survivors with sequelae.

Among 1970 children and young people $(<25$ years old) with IMD, there were 110 deaths $(5.6 \%)$ during index hospitalisation and 115 (5.8\%) deaths post-discharge. The distribution of cases and deaths in this young population is presented in Fig. 2.

Two survival curves were plotted for IMD cases and controls over the 6-year period. The first shows survival from the incident IMD episode, with all (acute phase and post-discharge) deaths, and the second shows post-discharge survival in those who survived the acute phase ('IMD survivors'), with only post-discharge deaths (Fig. 3).

Both survival curves showed significantly higher mortality $(p<0.0001)$ in IMD cases vs. controls, even for the smaller difference in deaths vs. controls post-discharge. IMD survivors were estimated to have a shorter life expectancy of 3.1 months on average vs. controls over the study period (i.e., average follow- 


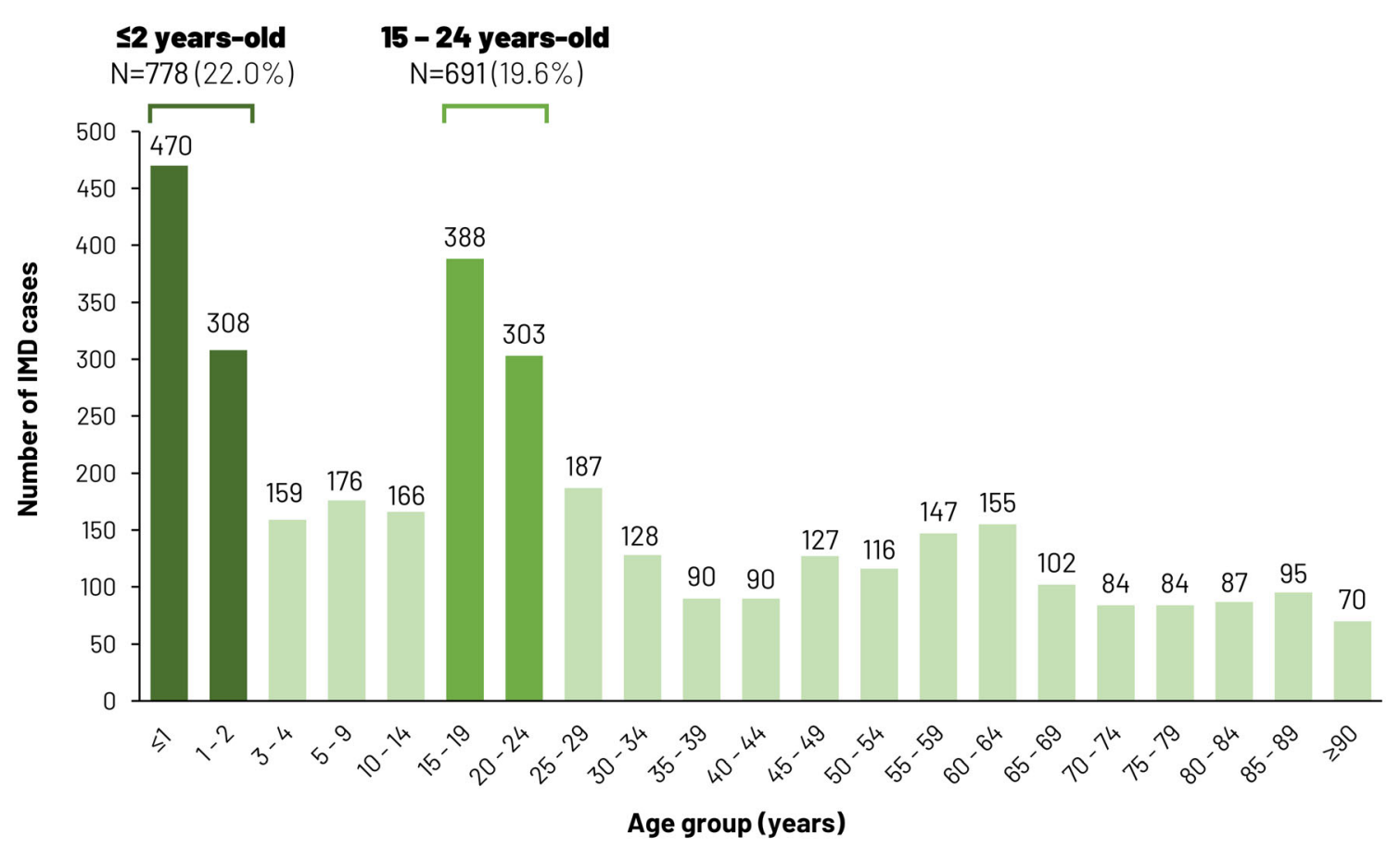

Fig. 1 IMD cases (all serogroups) over the study period by age group. $N$ number

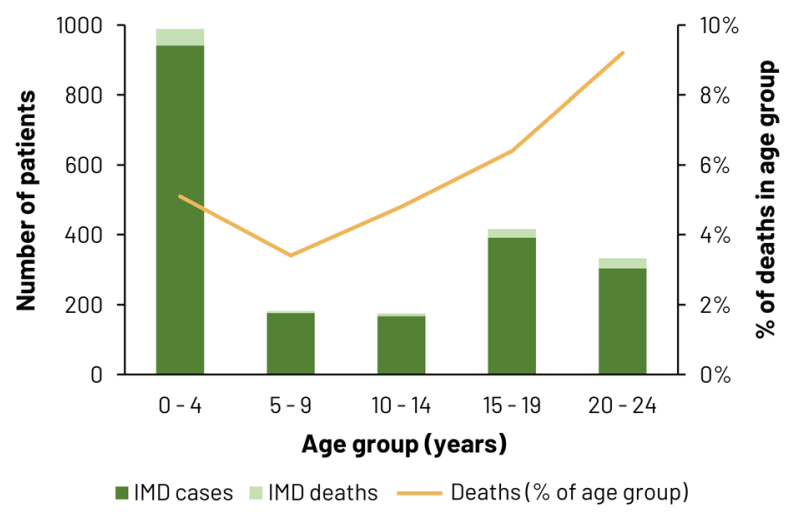

Fig. 2 Distribution of IMD cases and deaths (\%) (acute phase + post-discharge) aged $<25$ years

up $2.8 \pm 1.9$ years for IMD cases and $3.1 \pm 1.8$ years for controls).

One year after having IMD, the percentage of cases still alive was lower for cases who developed IMD at an older age, from $94.2 \%$ still alive for cases aged $<25$ years to $92.9 \%$ (25-49 years), $\quad 81.4 \% \quad$ (50-64 years), $\quad 81.3 \%$ (65-79 years) and $63.8 \%$ (> 79 years). KaplanMeier survival curves (including acute phase and post-discharge deaths) by age group $(<25$ years, $\quad 25-49$ years, 50-64 years,
65-79 years and $>79$ years) confirmed there was significantly higher mortality in IMD cases than controls in all age groups (all $p<0.0001$ ) and showed even greater differences in deaths post-discharge in age groups $>25$ years (see Supplementary Fig. 1). When considering only post-discharge survival curves in IMD survivors, the difference in deaths vs. controls remained statistically significant for incident IMD cases occurring at ages between 25 and 79 years old $(p<0.0001)$ (see Supplementary Fig. 2). Overall, after surviving the acute phase, IMD survivors of any age had an increased risk of death vs. controls (hazard rate [HR] 1.8 [95\% confidence interval (95\% CI) $1.5 ; 2.2])$. Figure 4 shows the highest observed HRs for post-discharge death in IMD survivors vs. controls were for ages between 25 and 60 years.

\section{Extrapolation of 6-Year Observed Data to Lifetime Life Expectancy}

After excluding cases who died during the index hospitalisation and their matched controls (as well as 43 remaining controls with a date of end of follow-up [death] prior to the date of 

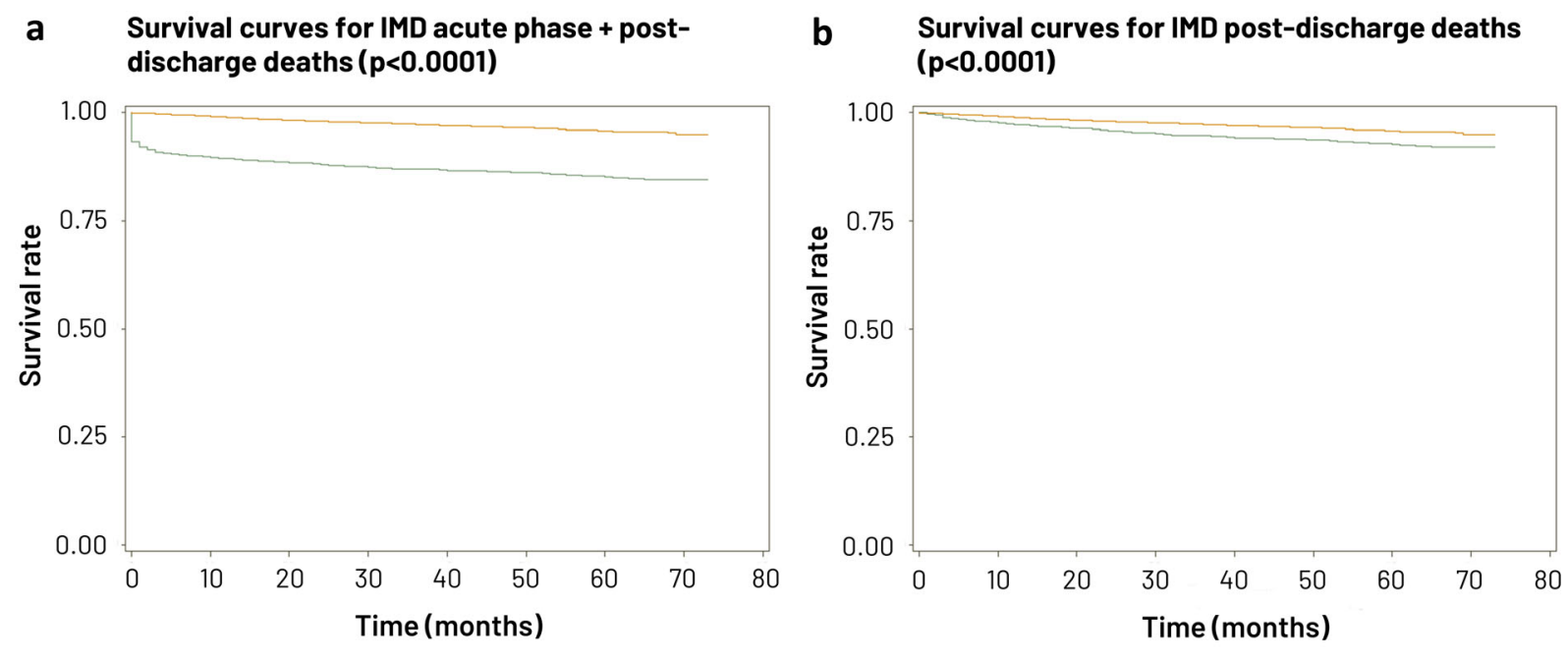

IMD cases Controls

Fig. 3 Survival curves for IMD cases and controls a from IMD episode in total population, b post-discharge in IMD survivors

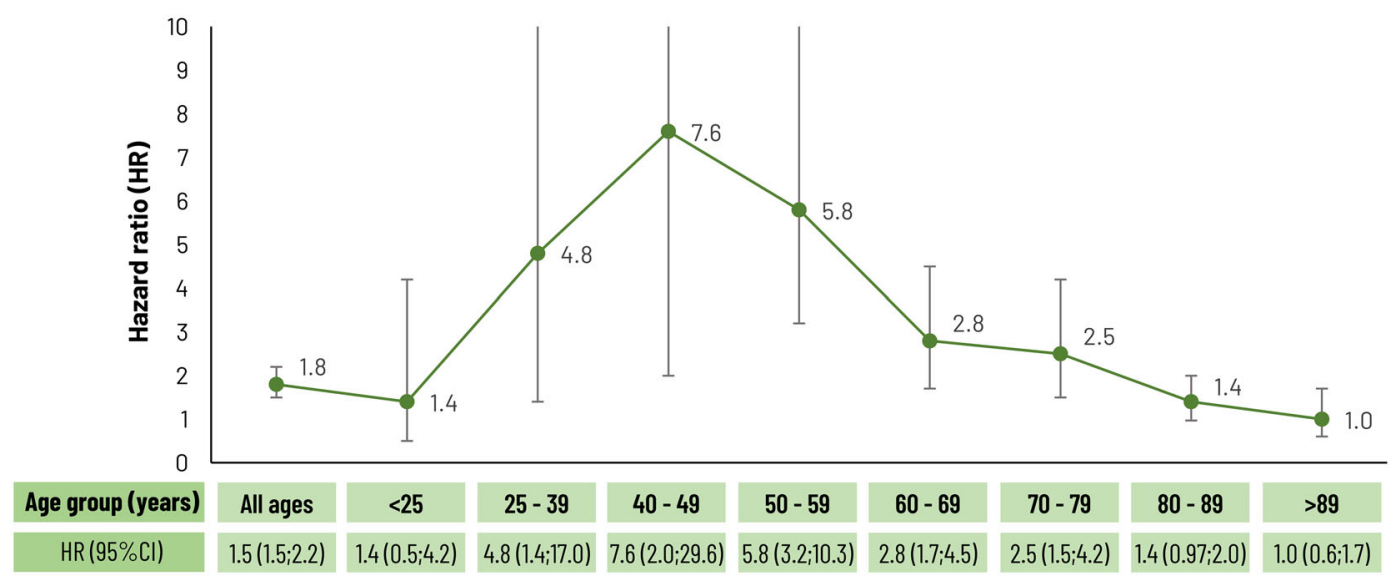

Fig. 4 Hazard rates (HR) for post-discharge death in IMD survivors vs. controls, by age group at infection. HR hazard rate, $I M D$ invasive meningococcal disease

inclusion [i.e., date of IMD diagnosis of their matched case]), there were 3239 IMD survivors and 9671 controls (total sample size of 12,910) for the extrapolation analysis.

Figure 5 shows the extrapolated survival curves for IMD survivors and controls. Figure 6 gives a visual presentation of expected life expectancy at age of IMD for the IMD survivors and matched controls. For example, infants $<1$ year with IMD who survived the acute phase of disease were predicted to live to
62.0 years on average compared to 79.3 years for controls.

\section{Need for State Financial Aid}

In France, a disability pension is paid to people to compensate for salary loss (SLC) due to a reduced working capacity as a result of an illness or accident of non-occupational origin. IMD cases were significantly more likely to receive a disability pension than controls $(1.0 \%$ of cases vs. $0.3 \%$ of controls, relative risk [RR] 3.9 [95\% 


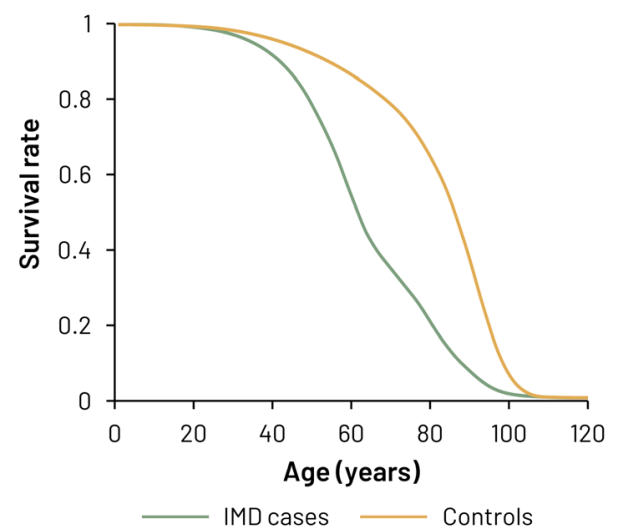

Fig. 5 Extrapolated lifetime survival curves and life expectancy by age at IMD

Life expectancy with:

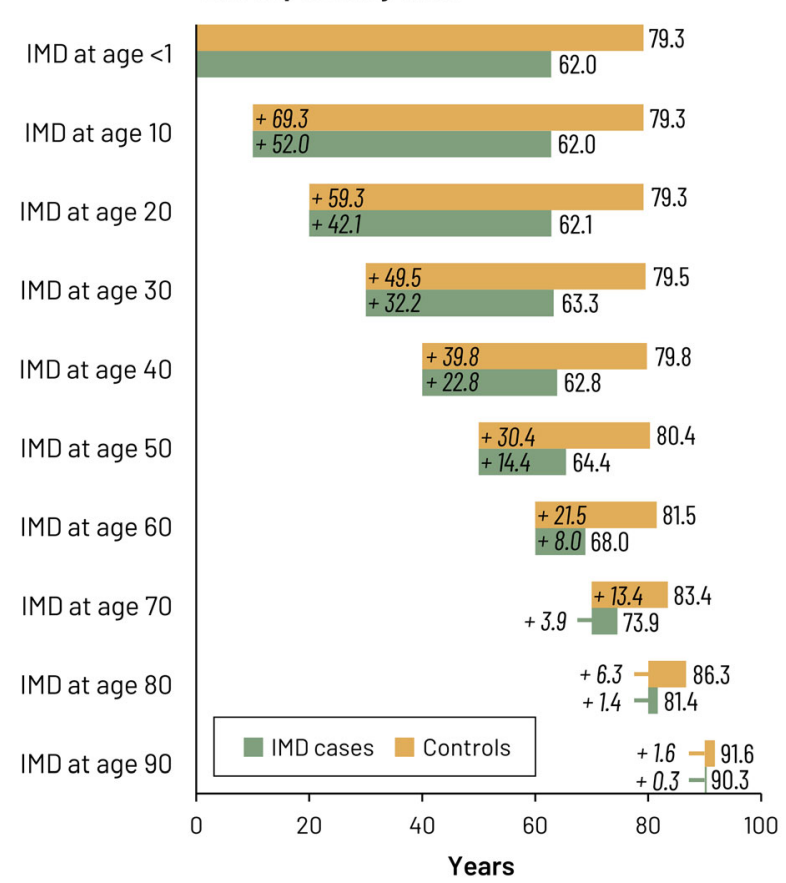

Fig. 6 Extrapolated lifetime life expectancy by age at IMD. $I M D$ invasive meningococcal disease

CI $2.3 ; 6.4])$ and at a lower average age than controls (44.3 years [standard deviation (SD) 11.2] vs. 51.2 years [SD 7.6]) (Table 1).

People with a long-term disease in France can benefit from exemption from co-payments for care and treatment related to the condition (ALD benefit), renewable every 5 years as applicable. IMD survivors were significantly more
Table 1 Demands for state financial aid in IMD cases and controls during acute phase and post-discharge

\begin{tabular}{|c|c|c|c|}
\hline & $\begin{array}{l}\text { IMD cases } \\
N=3532\end{array}$ & $\begin{array}{l}\text { Controls } \\
N=10,590\end{array}$ & $\begin{array}{l}\text { RR }(95 \% \\
\text { CI) } \\
{ }_{\text {*stat sig }}\end{array}$ \\
\hline \multicolumn{4}{|c|}{ Disability pension (SLC) } \\
\hline All ages & $35(0.99 \%)$ & $27(0.25 \%)$ & $\begin{array}{c}3.9(2.3 ; \\
6.4)^{*}\end{array}$ \\
\hline$<25$ years & 3 & 0 & $\begin{array}{c}89.9(0.2 ; \\
\quad 49,014.8)\end{array}$ \\
\hline$\geq 25$ years & 32 & 27 & $\begin{array}{c}3.6(2.1 \\
5.9)^{*}\end{array}$ \\
\hline
\end{tabular}

Long-term sickness (ALD)

$$
\begin{array}{lllc}
\text { All ages } & 243(6.88 \%) & 394(3.72 \%) & \begin{array}{c}
1.85(1.71 ; \\
2.00)^{*}
\end{array} \\
& & & 3.85(2.89 ; \\
<25 \text { years } & 108 & 84 & 5.14)^{*} \\
& & & 1.31(1.06 ; \\
\geq 25 \text { years } & 135 & 310 & 1.60)^{*}
\end{array}
$$

Complementary health insurance $(\mathrm{CMUc})$

$$
\begin{aligned}
& \text { All ages } \quad 195(5.52 \%) \quad 588(5.55 \%) \quad 0.99 \text { (0.84; } \\
& 1.17) \\
& \begin{array}{llll}
<25 \text { years } & 119 & 438 & 0.80(0.65 \text {; }
\end{array} \\
& 0.99) \\
& \geq 25 \text { years } 76 \quad 150 \quad 1.55(1.17 \text {; } \\
& 2.05)^{*}
\end{aligned}
$$

Note: Pre-existing state benefits (prior to IMD): 76 (2.2\%) of cases and $113(1.1 \%)$ of controls received SLC; 814 $(23.0 \%)$ of cases and 1384 (13.1\%) of controls received ALD; 587 (16.6\%) of cases and 1114 (10.5\%) of controls received CMUc

95\% CI 95\% confidence interval, $A L D$ affection de longue durée, $C M U c$ Couverture Médicale Universelle complémentaire; $I M D$ invasive meningococcal disease, $R R$ relative risk, $S L C$ salary loss compensation (Pension d'invalidité)

${ }^{*}$ indicates statistically significant values

likely to receive ALD benefits compared with controls $(6.9 \%$ of cases vs. $3.7 \%$ of controls, RR 1.85 [95\% CI 1.71; 2.00]) (Table 1). Among those without pre-existing ALD benefits, 8.9\% of IMD cases vs. $4.3 \%$ of controls claimed new 
ALD benefits following IMD hospitalisation. The majority of ALD beneficiaries in the control group were aged 60 years and older $(53.0 \%$ vs. $28.4 \%$ among IMD cases), while $44.4 \%$ of IMD cases with ALD benefits were aged under 25 years (vs. 21.3\% among controls). Of the 30 conditions for which ALD was claimed, six conditions were reported significantly more frequently in IMD cases vs. controls: haemophilia and haemostasis disorders (RR 33.0 [4.3; 255.4]), severe primary immune deficiency/human immunodeficiency virus (RR 8.5 [3.4; 21.5]), debilitating stroke (RR 2.0 [1.2; 3.3]), polyarteritis nodosa/systemic lupus erythematosus/generalised progressive scleroderma (RR 3.5 [1.2; 10.4]), chronic active liver disease and cirrhosis (RR $2.5[1.1 ; 5.8]$ ) and severe neurological and muscular disorders (including myopathy)/severe epilepsy (RR 1.9 [1.0; 3.9]).

People with a low income who are not covered by a compulsory health insurance scheme are eligible for the free complementary health insurance plan (CMUc), which reimburses medical expenses. Receiving CMUc benefits is an indicator of socioeconomic status. Prior to having IMD, $17.0 \%$ of cases vs. $10.5 \%$ of controls had claimed CMUc benefits $(p<0.0001)$. The proportion of CMUc beneficiaries among cases was significantly higher for all age groups, except for 15-24 year olds, where there was no difference between cases and controls. During the index IMD hospitalisation, $1.7 \%$ of cases vs. $0.2 \%$ of controls made a first CMUc claim compared with $4.0 \%$ of cases vs. $5.4 \%$ of controls during the post-discharge follow-up (of maximum 6 years). Thus, the overall differences in CMUc benefits due to IMD in the acute and post-discharge phase during the study period were not significantly different for cases and controls (RR 0.99 [95\% CI $0.84 ; 1.17]$ ) (Table 1).

\section{DISCUSSION}

The study used the SNIIRAM database to examine the impact of IMD on long-term mortality and the need for financial support from the state in France. The study showed that IMD not only has high mortality $(8.3 \%)$ during the acute phase, but also leads to a significantly reduced life expectancy following the index hospitalisation during the whole study period, regardless of age at which IMD occurred and regardless of sequelae. Overall, sequelae were identified in the database in $23.3 \%$ of IMD cases, and the risk of post-discharge death in IMD cases was observed to be $>2.5$ times higher in IMD cases with sequelae than in those without sequelae $(8.4 \%$ vs. $3.5 \%)$. The observed risk of sequelae in nearly a quarter of cases was comparable to findings from a German database study [14] but lower than expected ranges were reported by a case-control study in the UK (e.g., up to $30 \%$ in IMD survivors [6]), possibly because less visible sequelae (e.g., psychological and behavioural sequelae) may not be completely captured in the database or within the study time frame. The impact on life expectancy was expected to persist, e.g., IMD occurring at ages $0-50$ years was predicted to reduce lifetime life expectancy in survivors by around 16 years compared with controls. The study also found that IMD survivors are more likely to claim financial state support in terms of a disability pension (RR 3.9 [95\% CI 2.3; 6.4]) and exemption from healthcare costs due a long-term condition (RR 1.85 [95\% CI 1.71; 2.00]), though the increase in demand for CMUc benefits following IMD was comparable to that in controls (RR 0.99 [95\% CI 0.84; 1.17]). The SNIIRAM database provides an extensive and rich source of information covering nearly the entire French population, and the use of real-world data provides strong evidence highlighting the long-term burden and economic impact of IMD in France.

Only three studies were identified in the literature assessing long-term mortality risk in IMD survivors; all were based on a national Danish database [21-23]. The first Danish study, published in 1986, on 782 IMD survivors diagnosed between 1966 and 1976 found that 11\% died during the 4-15-year follow-up, and late excess mortality rates (particularly in the first 2 years following meningitis) were significantly higher compared to matched expected general population rates, especially among 30-60 year olds and more severe initial cases [21]. The second Danish study of 2902 childhood/ 
adolescent IMD cases diagnosed from 1980 to 2009 followed 1028 patients until the age of 30 . Although patient mortality immediately following disease diagnosis was higher in cases than controls, the study found comparable mortality rates in survivors until age 30 who received adequate treatment compared to the general population [22]. A third Danish study of all 4909 IMD patients diagnosed from 1977 to 2006 and alive 1 year later found that IMD is associated with a small but increased risk of long-term mortality compared to the general population (mortality rate ratio MRR 1.27 [95\% CI 1.12-1.45]). The increased mortality risk persisted throughout the study period with no differences by sex and was statistically significant by age group for patients aged $>30$ years at IMD diagnosis. The risk of death in IMD survivors was especially high due to nervous system diseases (MRR 3.57 [95\% CI 1.82-7.00]) [23]. The two Danish studies on all age IMD cases found an increased long-term mortality risk, especially in cases where IMD occurred after age 30 years. This is similar to the observed data with up to 6-years follow-up in the SNIIRAM database, showing that post-discharge survival in IMD survivors remained statistically significantly lower than in controls for incident IMD cases occurring at ages between 25 and 79 years old $(p<0.0001)$. The Danish study in IMD cases occurring in childhood or adolescence observed no long-term increased mortality risk when cases were observed until age 30 years. However, our analysis found that in France, when data were extrapolated to lifetime, there was a significant impact on life expectancy, even for childhood and adolescent cases, which was similar to the reduction predicted for older cases.

A database analysis in Germany assessed long-term IMD direct medical costs with up to 7-year follow-up, but only assessed IMD mortality with up to 1-year follow-up. IMD cases diagnosed from 2009 to 2015 and controls were assessed from the German database InGef (which includes inpatient and outpatient claims data from statutory health insurance providers). The 30-day mortality was $4.3 \%$ (95\% CI $1.7-8.6 \%)$ and the 1-year mortality was $5.5 \%$ (95\% CI 2.5-10.2\%) [14]. These mortality data are likely primarily due to mortality in the acute phase (i.e., index hospitalisation), given the time frame of evaluation, but are somewhat lower than the acute phase mortality in France in our analysis (although our point estimate of 8.3\% falls within the German 95\% confidence intervals).

The long-term economic cost of IMD to the healthcare system, society or patients has not been widely studied in France. A recent publication estimated the lifetime cost based on two realistic hypothetical cases of severe IMD in young children. They estimated the life expectancy to be 77 years and 55 years, respectively, and the lifetime costs to range from $€ 768,875$ to $€ 1,480,546$ for the first case and from $€ 1,924,475$ to $€ 2,267,251$ for the second case in France. These cases highlighted that the outcomes of IMD can be diverse and result in a lifelong need for healthcare provision, in addition to having an impact on education and work [10]. A healthcare cost analysis based on this data set has recently been published and confirms the high medical costs of IMD in France, i.e., with a mean cost for the index hospitalisation of $€ 11,256$ and subsequent annual costs increasing to over $€ 20,000$ in cases with two or more sequelae [24]. Our study has shown that even during the maximum 6-year observed follow-up period, IMD cases are much more likely to claim state benefits to cover longterm healthcare costs or lost income due to disability, and from a younger average age than the general population. This suggests that IMD cases may be dependent on the state for financial aid for a longer period than the general population, as several IMD sequelae can persist over the patients' lifetime.

Our analysis showed that having a long-term illness and receiving ALD benefits was a risk factor for IMD hospitalisation. Following IMD hospitalisation, among those without previous ALD benefits, however, $8.9 \%$ of IMD cases vs. $4.3 \%$ of controls claimed new ALD benefits. The database analysis shows that six ALDs were significantly more frequently claimed among IMD cases than controls; however, it is not possible from the data available to determine whether these were related to worsening of pre-existing conditions or to the development of new 
conditions following IMD. Some sequelae, such as severe neurological and muscular disorders (including myopathy and severe epilepsy), are already well-recognised sequelae of IMD. A recent systematic review of the literature has identified a range of 44 different sequelae that can result following IMD [25], including physical, neurological and psychological or behavioural conditions [15].

A previously published analysis of this IMD data set found that, prior to IMD, there was an association between CMUc status (an indicator of lower socioeconomic status) and risk of IMD hospitalisation, particularly in infants (OR 4.81 [95\% CI 3.56; 6.49]) and cases aged $>25$ years [17]. This analysis found an increase in new claims for CMUc during or following IMD (around 5.5\%); however, this was comparable to the control group. The previously published risk factor analysis [17] suggests that IMD hospitalisation is associated with medical risk factors as well as socioeconomic risk factors (such as CMUc status, which is a proxy for low income). The present analysis has shown that following IMD, survivors are also at increased risk of further health inequity such as having a greater likelihood of requiring ALD and SLC support. A national immunization programme (NIP) ensures access to vaccination for all, regardless of socioeconomic status or other medical risk factors. An NIP can, therefore, help to reduce socioeconomic inequity that increases the risk of disease and health inequity that follows disease in all vulnerable populations. This study shows that the financial impact of IMD goes beyond costs to the healthcare sector, and suggests that to fully capture the benefits and costeffectiveness of preventive interventions (e.g., meningococcal vaccinations) a broad societal perspective is needed.

The findings of this study may have implications for other European countries where IMD follows similar trends (i.e., comparative incidence, serogroup B predominance, cases occurring in all age groups with a higher incidence in infants, children and adolescents [3]) and where countries have a welfare or social security system in place to ensure patients with chronic and long-term conditions are cared for [26, 27], e.g., as in France, with the provision of state financial aid. Real-world databases may be increasingly used to understand the impact of IMD, or other uncommon diseases, and as a useful resource to inform policymakers regarding future needs of patients with long-term conditions.

The extensive disease burden of IMD and long-lasting economic consequences highlight the need for disease prevention. As the predominant serogroups causing disease continue to change, IMD epidemiology remains unpredictable. As such, disease prevention through vaccination should be continued and even extended to ensure the prevention of IMD from all serogroups. The SNIIRAM database did not report IMD cases by the Neisseria meningitidis serogroup causing disease. During the study period (2012-2017) and for at least the last 30 years in France, serogroup B has been the predominant serogroup causing IMD. In 2017, serogroup B infection was responsible for around $60 \%$ of IMD in infants and children aged 1-14 years, around 40\% of IMD in adolescents and adults and around 20\% of IMD in adults aged $\geq 60$ years [28].

A key strength of this study was being able to capture a large IMD population with sufficient details regarding all contacts with health services, in and out of hospital, providing previously unknown data in France on the lifetime impact of IMD. This study also had several limitations. The full duration of follow-up of the study was 6 years (from 2012 to 2017), and the average duration of follow-up for the survival analysis was around 3 (range 1 to 6) years, as patients were followed until death or the study end date. This is a limitation of the analysis, and having a longer follow-up period would be beneficial in future studies to add more certainty to the long-term survival extrapolation findings. IMD cases were identified by ICD-10 codes, which do not provide additional details, e.g., about serogroup of IMD; therefore, all serogroups of IMD cases were analysed as a whole. IMD cases who were not hospitalised were also not captured by the database, e.g., cases who may have died before seeking medical care. IMD is known to result in a high mortality rate within $24 \mathrm{~h}$ of first symptoms [29]. While this study was able to capture 
financial claims due to long-term health conditions following IMD, the full economic impact of the disease over the lifetime of the patient is much broader, affecting non-health areas not covered in the database, e.g., the impact on ability to work of patients and their family members who may have to care for them on the long term. Even so, the findings clearly show that IMD burden goes beyond the healthcare sector and warrants a broader societal perspective when assessing the benefits of preventive interventions.

\section{CONCLUSION}

IMD is known to result in high mortality during the acute phase, but has now also been shown to lead to reduced life expectancy following the index hospitalisation, regardless of sequelae. Furthermore, this impact is likely to persist, as shown in the extrapolation of mortality over lifetime. In France, the financial burden extends beyond the healthcare sector, as IMD survivors are also more likely to claim state financial support, such as salary loss compensation due to disability and benefits to cover healthcare costs for a long-term condition. The broad burden of IMD highlights the need for prevention of the disease, for example, through vaccination programmes.

\section{ACKNOWLEDGEMENTS}

Funding. GlaxoSmithKline Biologicals SA funded this study and all cost related to the development and publication of this manuscript, including journal's Rapid Service Fee and assistance.

Medical Writing, Editorial, and Other Assistance. The authors thank Business and Decision Life Sciences plateform for editorial assistance and publication coordination, on behalf of GSK. Pierre-Paul Prévot coordinated publication development and editorial support. Kavi Littlewood (Littlewood Writing Solutions) provided medical writing support. The authors acknowledge the contributions of Mohamed Neine (freelance), Muhamed-Kheir Taha (external expert, Institut Pasteur), Catherine WeilOlivier (external expert, Université Paris VII), Céline Pribil, Gaëlle Nachbaur and Valérie Grange (GSK) for their participation in the scientific committee and involvement in the discussions.

Authorship. All named authors meet the International Committee of Medical Journal Editors (ICMJE) criteria for authorship for this article, take responsibility for the integrity of the work as a whole, and have given their approval for this version to be published.

Author Contribution. All authors contributed to conceptualisation, methodology, analysis, drafting and review of the present work.

Disclosures. Emmanuel Aris and Ekkehard Beck are employees of and held shares in the GSK group of companies. Jing Shen was employee of and hold shares in the GSK group of companies during the study. Jing Shen's current affiliation is Takeda Pharmaceutical Company Limited, Zurich, Switzerland. Stéphane Bouée and Corinne Emery declare that their institution (CEMKA) received fees from the GSK group of companies for the present study. Authors declare no other financial and non-financial relationships and activities.

Compliance with Ethics Guidelines. The study was conducted in accordance with the Helsinki Declaration of 1964 and later amendments, and relevant international and French regulatory requirements. Patient data in the database are anonymised using an irreversible double encryption. Access to the SNIIRAM database is regulated by a Committee of Expertise for Research, Studies and Evaluations in the field of Health, to which the present study protocol was submitted for approval. Since this was a retrospective study of an anonymised database and had no influence on patient care, ethics committee approval was not required. Use of the database for this type of study is regulated by the French national data 
protection agency (Commission Nationale de l'Informatique et des Libertés), to which the protocol was submitted for approval.

Data Availability. The datasets generated during and/or analysed during the current study are not publicly available due to the access to the data is restricted for a limited time period due to French regulations. The study was conducted in accordance with the Helsinki Declaration of 1964, and its later amendments, as well as with relevant international and French regulatory requirements. Patient data in the database are anonymised using an irreversible double encryption. Access to the SNDS is regulated by a Committee of Expertise for Research, Studies and Evaluations in the field of Health (CEREES), to which the present study protocol was submitted for approval. Use of the SNDS database for this type of study is regulated by the French national data protection agency (Commission Nationale de l'Informatique et des Libertés), to which the protocol was submitted for approval.

Open Access. This article is licensed under a Creative Commons Attribution-NonCommercial 4.0 International License, which permits any non-commercial use, sharing, adaptation, distribution and reproduction in any medium or format, as long as you give appropriate credit to the original author(s) and the source, provide a link to the Creative Commons licence, and indicate if changes were made. The images or other third party material in this article are included in the article's Creative Commons licence, unless indicated otherwise in a credit line to the material. If material is not included in the article's Creative Commons licence and your intended use is not permitted by statutory regulation or exceeds the permitted use, you will need to obtain permission directly from the copyright holder. To view a copy of this licence, visit http:// creativecommons.org/licenses/by-nc/4.0/.

\section{REFERENCES}

1. Nadel S, Ninis N. Invasive meningococcal disease in the vaccine era. Front Pediatr. 2018. https://doi.org/ 10.3389/fped.2018.00321.

2. World Health Organization (WHO) Meningitis. https://www.who.int/health-topics/ meningitis\#tab=tab_1. (accessed Mar 17, 2021)

3. European Centre for Disease Prevention and Control (ECDC) Factsheet about meningococcal disease. https://www.ecdc.europa.eu/en/meningococcaldisease/factsheet. (accessed Mar 17, 2021)

4. Wang B, Santoreneos R, Giles L, et al. Case fatality rates of invasive meningococcal disease by serogroup and age: a systematic review and metaanalysis. Vaccine. 2019;37:2768-82. https://doi. org/10.1016/j.vaccine.2019.04.020.

5. World Health Organization (WHO) FactsheetMeningococcal meningitis. 2018. https://www. who.int/news-room/fact-sheets/detail/ meningococcal-meningitis (accessed Mar 17, 2021)

6. Viner RM, Booy R, Johnson H, et al. Outcomes of invasive meningococcal serogroup $B$ disease in children and adolescents (MOSAIC): a case-control study. Lancet Neurol. 2012;11:774-83. https://doi. org/10.1016/s1474-4422(12)70180-1.

7. Martinón-Torres F. Deciphering the burden of meningococcal disease: conventional and underrecognized elements. J Adolesc Health. 2016;59: S12-20. https://doi.org/10.1016/j.jadohealth.2016. 03.041 .

8. Peterson ME, Li Y, Bita A, et al. Meningococcal serogroups and surveillance: a systematic review and survey. J Glob Health. 2019;9:010409-09. https://doi.org/10.7189/jogh.09.010409.

9. Anonychuk A, Woo G, Vyse A, et al. The cost and public health burden of invasive meningococcal disease outbreaks: a systematic review. Pharmacoeconomics. 2013;31:563-76. https://doi.org/10. 1007/s40273-013-0057-2.

10. Bénard S, Wright C, Voisine J, et al. Lifetime cost of meningococcal disease in France: scenarios of severe meningitis and septicemia with purpura fulminans. J Infect Public Health. 2016;9:339-47. https:// doi.org/10.1016/j.jiph.2015.10.016.

11. European Centre for Disease Prevention and Control (ECDC). Vaccine scheduler-meningococcal disease: recommended vaccinations. 2021. https:// vaccine-schedule.ecdc.europa.eu/Scheduler/ ByDisease?SelectedDiseaseId= 
48\&SelectedCountryIdByDisease=-1. (accessed Mar $25,2021)$

12. UK National Health Service Vaccination - Meningitis. 2021. https://www.nhs.uk/conditions/ meningitis/vaccination/\#: :text=The $\%$ 20meningitis\%20B\%20vaccine $\% 20$ is, $\mathrm{a} \%$ 20booster\%20at\%201\%20year. (accessed Mar 25, 2021)

13. Taha MK, Gaudelus J, Deghmane AE, et al. Recent changes of invasive meningococcal disease in France: arguments to revise the vaccination strategy in view of those of other countries. Hum Vaccin Immunother. 2020;16:2518-23. https://doi.org/10. $1080 / 21645515.2020 .1729030$.

14. Huang L, Heuer OD, Janßen S, et al. Clinical and economic burden of invasive meningococcal disease: evidence from a large German claims database. PLoS One. 2020;15: e0228020. https://doi. org/10.1371/journal.pone.0228020.

15. Olbrich KJ, Müller D, Schumacher S, et al. Systematic review of invasive meningococcal disease: sequelae and quality of life impact on patients and their caregivers. Infect Dis Ther. 2018;7:421-38. https://doi.org/10.1007/s40121-018-0213-2.

16. Bezin J, Duong M, Lassalle R, et al. The national healthcare system claims databases in France, SNIIRAM and EGB: powerful tools for pharmacoepidemiology. Pharmacoepidemiol Drug Saf. 2017;26:954-62. https://doi.org/10.1002/pds.4233.

17. Taha MK, Weil-Olivier C, Bouée S, et al. Risk factors for invasive meningococcal disease: a retrospective analysis of the French national public health insurance database. Hum Vaccin Immunother. 2021. https://doi.org/10.1080/21645515.2020. 1849518.

18. Scholz S, Koerber F, Meszaros K, et al. The cost-ofillness for invasive meningococcal disease caused by serogroup B Neisseria meningitidis (MenB) in Germany. Vaccine. 2019;37:1692-701. https://doi. org/10.1016/j.vaccine.2019.01.013.

19. Beck E, Klint J, Neine M, et al. Cost-effectiveness of 4CMenB infant vaccination in England: a comprehensive valuation considering the broad impact of serogroup $B$ invasive meningococcal disease. Value Health. 2021;24:91-104. https://doi.org/10.1016/j. jval.2020.09.004.

20. Royston P, Lambert PC. Flexible parametric survival analysis using stata: beyond the cox model. College Station: StataCorp LP; 2011.
21. Kjersem H, Bohr V, Rasmussen N, et al. Mortality in the years following bacterial meningitis. Infection. 1986;14:55-9. https://doi.org/10.1007/bf01644442.

22. Pickering $\mathrm{L}$, Jennum $\mathrm{P}$, Ibsen $\mathrm{R}$, et al. Long-term health and socioeconomic consequences of childhood and adolescent onset of meningococcal meningitis. Eur J Pediatr. 2018;177:1309-15. https://doi.org/10.1007/s00431-018-3192-0.

23. Roed C, Omland LH, Engsig FN, et al. Long-term mortality in patients diagnosed with meningococcal disease: a Danish nationwide cohort study. PLoS One. 2010;5: e9662. https://doi.org/10.1371/ journal.pone.0009662.

24. Weil-Olivier C, Taha M-K, Emery C, et al. Healthcare resource consumption and cost of invasive meningococcal disease in France: a study of the National Health Insurance Database. Infect Dis Ther. 2021;10:1607-23. https://doi.org/10.1007/ s40121-021-00468-w.

25. Shen J, Begum N, Ruiz Garcia Y, et al. PD051/\#550: the long-lasting impact of invasive meningococcal disease (IMD) - mapping the comprehensive range of sequelae in imd survivors. 2021. European Society for Paediatric Infectious Diseases Geneva, Switzerland

26. Nolte E, Knai C, Saltman RB. Assessing chronic disease management in European health systems. Assessing chronic disease management in European health systems: Concepts and approaches. European Observatory Health Policy Series. Copenhagen (Denmark): European Observatory on Health Systems and Policies (c) World Health Organization 2014 (acting as the host organization for, and secretariat of, the European Observatory on Health Systems and Policies). 2014

27. European Association of Service Providers for Persons with Disabilities (EASPD) Social welfare systems across Europe. https://www.easpd.eu/sites/ default/files/sites/default/files/SensAge/d4-social welfare_systems_across_europe.pdf. (accessed May $25,2021)$

28. Sante Publique France Les infections invasives à méningocoque en France en 2017 2017. https:// www.santepubliquefrance.fr/maladies-ettraumatismes/maladies-a-prevention-vaccinale/ infections-invasives-a-meningocoque/documents/ bulletin-national2/les-infections-invasives-ameningocoque-en-france-en-2017. (accessed Sep $14,2021)$ 
29. Beebeejaun K, Parikh SR, Campbell H, et al. Invasive meningococcal disease: timing and cause of death in England, 2008-2015. J Infect. 2020;80:286-90. https://doi.org/10.1016/j.jinf.2019.12.008.

\section{Publisher's Note}

Springer Nature remains neutral with regard to jurisdictional claims in published maps and institutional affiliations. 